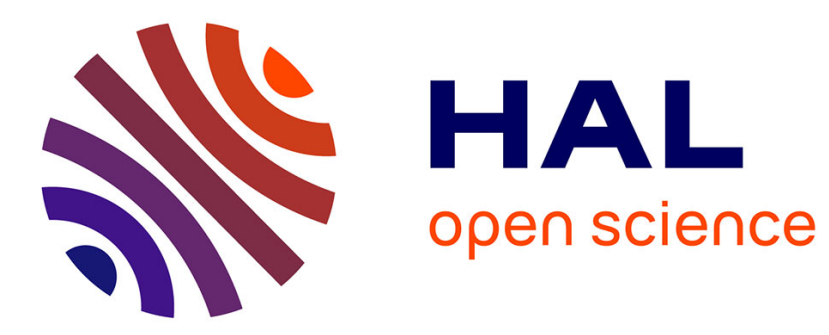

\title{
A New Characterization of Mobility for Distance-Bearing Formations of Unicycle Robots
}

Fabio Morbidi, Estelle Bretagne

\section{To cite this version:}

Fabio Morbidi, Estelle Bretagne. A New Characterization of Mobility for Distance-Bearing Formations of Unicycle Robots. IEEE/RSJ International Conference on Intelligent Robots and Systems, Oct 2018, Madrid, Spain. pp.4833-4839. hal-01891511

\section{HAL Id: hal-01891511 \\ https://hal.science/hal-01891511}

Submitted on 9 Oct 2018

HAL is a multi-disciplinary open access archive for the deposit and dissemination of scientific research documents, whether they are published or not. The documents may come from teaching and research institutions in France or abroad, or from public or private research centers.
L'archive ouverte pluridisciplinaire HAL, est destinée au dépôt et à la diffusion de documents scientifiques de niveau recherche, publiés ou non, émanant des établissements d'enseignement et de recherche français ou étrangers, des laboratoires publics ou privés. 


\title{
A New Characterization of Mobility for Distance-Bearing Formations of Unicycle Robots
}

\author{
Fabio Morbidi, Estelle Bretagne
}

\begin{abstract}
In this paper, we present a new characterization of mobility for formations of unicycle robots defined by distance-bearing constraints. In fact, by introducing a simple reduction procedure which associates a prescribed formation with a "macro-robot", we extend the classification by type proposed by Campion et al., to multi-agent systems. To simplify the classification task, which only leverages the nonslip condition for a conventional centered wheel, we assume that the robots are disposed at the vertices of a regular convex polygon. We demonstrate the practical utility of the notion of macrorobot in a trajectory-tracking control problem for a formation of unicycles.
\end{abstract}

\section{INTRODUCTION}

Teams of cooperating robots are being increasingly deployed in the real world for complex tasks, such as object transport [1], warehouse management [2], and inspection of industrial infrastructures [3], to name but a few relevant examples. However, while considerable efforts have gone into the design of (distributed) algorithms for controlling the geometric shape of multi-robot formations [4], [5], relatively few works [6], [7], so far, have dealt with their structural properties. Notably, a general and comprehensive theory which explains how the formation constraints affect the mobility of the resulting multi-robot system is, to the best of our knowledge, still missing. A vast literature on distance rigidity [8], [9] and more recently on bearing and $\operatorname{SE}(2)$ rigidity [10]-[13] of robot formations has emerged, but it has only provided a partial answer to the motion feasibility problem. In fact, simplistic mathematical models (single or double integrators) have been considered for the vehicles therein, and the constraints resulting from wheel-ground interaction have been overlooked.

The characterization of mobility of wheeled mobile robots (WMRs) described by kinematic and dynamic models, and their elegant classification into five types according to their degrees of mobility and steerability, traces back to the seminal work of Campion et al. [14] in the 90's. The nonslip condition, i.e. the fact that the kinematic constraints imply that the velocity of the center of a conventional wheel is parallel to the wheel plane, plays a crucial role in [14]. Our goal in this paper is to revisit and extend the classification by type in [14] to formations of unicycle robots specified by relative distance and bearing constraints. To simplify our analysis, we decided to turn our attention to robots with conventional centered orientable wheels disposed at the vertices of regular convex polygons. While this might seem a serious restriction, this class of formations is extremely rich and diverse,

The authors are with the MIS laboratory, Université de Picardie Jules Verne, 33 rue Saint-Leu, 80039 Amiens, France. Emails: ffabio.morbidi, estelle.bretagne\}@u-picardie.fr since a large variety of distance-bearing constraints can be imposed. Moreover, the members of this class fall into few generic categories inherited from the single-vehicle case and independent of robots' number. We avail ourselves of a simple reduction procedure, leading to the definition of a "macro-robot" (or m-robot, in short): the "wheels" of the $\mathrm{m}$-robot correspond to the original vehicles in the formation, and the geometry of its "chassis" depends on the nature of the distance-bearing constraints imposed on the robots. The nonslip condition can be defined for each "wheel" of the m-robot, from which the degree of mobility and steerability, and thus the formation type can be deduced using the tools developed in [14]. The taxonomy proposed in this paper is not merely of theoretical interest. In fact, it may inform the design of more efficient formation control strategies for multiple nonholonomic robots and shed some light on the intrinsic limitations of the existing ones (see [15]-[18] and the references therein). In particular, thanks to our reduction procedure, we could easily adapt the trajectorytracking controller via dynamic feedback originally proposed in [19] for mobile robots with multiple steering wheels, to distance-bearing formations of unicycles.

It is finally worth pointing out here that an early attempt towards a characterization of the mobility of leader-follower formations of unicycles was made in [20]. However, the analysis in [20] departs from ours, since it does not leverage the nonslip condition for centered wheels, but the more abstract, system-theoretic notion of internal dynamics.

The rest of this paper is organized as follows. In Sect. II, we briefly review the classification of WMRs by type proposed in [14]. In Sect. III, this classification is extended to distance-bearing formations of unicycle robots. In Sect. IV, we discuss the results of numerical simulations featuring a multi-robot formation tracking a reference trajectory, and in Sect. V some possible directions for future research are outlined.

Notation: The symbol $\mathbb{Z}$ indicates the set of integers, $\mathbb{R}^{m \times n}$ the space of all $m \times n$ real matrices, and

$$
\mathbf{R}(\theta)=\left[\begin{array}{ccc}
\cos \theta & -\sin \theta & 0 \\
\sin \theta & \cos \theta & 0 \\
0 & 0 & 1
\end{array}\right],
$$

the elementary rotation matrix of an angle $\theta$ about the $z$-axis. $\forall \mathbf{x}, \mathbf{y} \in \mathbb{R}^{n},\langle\mathbf{x}, \mathbf{y}\rangle=\sum_{i=1}^{n} x_{i} y_{i}$, and $\|\mathbf{x}\|=\langle\mathbf{x}, \mathbf{x}\rangle^{1 / 2}$. $\boldsymbol{f}(t) \in \mathcal{C}^{k}$ means that the vector-valued function $\boldsymbol{f}(t)$ is of differentiability class $\mathcal{C}^{k}$. Finally, $\mathbf{I}_{n \times n}$ and $\mathbf{0}_{m \times n}$ denote the $n \times n$ identity matrix and the $m \times n$ matrix of zeros, respectively, and $\operatorname{nul}(\mathbf{A})$ the nullity of matrix $\mathbf{A} \in \mathbb{R}^{m \times n}$, i.e. the dimension of the kernel of $\mathbf{A}$. 


\section{MobILITY OF WHEELED ROBOTS}

In this section we will briefly review the standard classification of WMRs by type [14], [21]. We will start by introducing the nonslip condition for a conventional centered wheel, and proceed to define the degree of mobility and steerability of a robot. Let $\left\{O_{I} ; x_{I}, y_{I}\right\}$ be an inertial frame, and $\left\{O_{R} ; x_{R}, y_{R}\right\}$ the moving frame rigidly attached to a robot (see Fig. 1). The pose of the robot with respect to the inertial frame, is described by the posture vector $\mathbf{q}=[x, y, \theta]^{T}$, where $x$ and $y$ are the coordinates of point $O_{R}$ and $\theta$ describes the orientation of the moving frame with respect to the inertial frame. The position of the center of the wheel $A$ is expressed in polar coordinates by the distance $\ell$ and angle $\alpha$. Finally, the angle between the segment $\overline{O_{R} A}$ and the axle of the wheel (a.k.a. propulsion axis) is denoted by $\beta$. For a conventional wheel, the kinematic constraints imply that the velocity of the center of the wheel is parallel to the wheel plane (nonslip condition) and proportional to the wheel spinning velocity (pure rolling condition). For a centered (passive or active) wheel, the nonslip condition at the contact point between the wheel and the ground reads,

$$
[\cos (\alpha+\beta), \sin (\alpha+\beta), \ell \sin \beta] \mathbf{R}^{T}(\theta) \dot{\mathbf{q}}=0,
$$

where $\mathbf{R}^{T}(\theta) \dot{\mathbf{q}}$ is used to transform the motion parameters $\dot{\mathbf{q}}$ expressed in the inertial reference frame, into motion parameters in the local reference frame of the robot. For a fixed wheel, $\beta$ in (1) is constant, while for a steering wheel, $\beta=\beta(t)$, i.e. it varies as a function of time (being the wheel actuated or non-actuated). Let us now consider a robot equipped with $n$ centered wheels. It is expedient to introduce the subscripts " $f$ " and " $s$ " to identify quantities related to fixed and steering wheels, respectively. The number of wheels of each type are denoted by $n_{f}$ and $n_{s}$, with $n=n_{f}+n_{s}$. The nonslip conditions for centered fixed or steering wheels can be rewritten in a compact form as,

$$
\mathbf{C}_{1}^{*}\left(\boldsymbol{\beta}_{s}\right) \mathbf{R}^{T}(\theta) \dot{\mathbf{q}}=\mathbf{0},
$$

where $\mathbf{C}_{1}^{*}\left(\boldsymbol{\beta}_{s}\right)=\left[\mathbf{C}_{1 f}^{T}, \mathbf{C}_{1 s}^{T}\left(\boldsymbol{\beta}_{s}\right)\right]^{T}, \mathbf{C}_{1 f} \in \mathbb{R}^{n_{f} \times 3}$, $\mathbf{C}_{1 s}\left(\boldsymbol{\beta}_{s}\right) \in \mathbb{R}^{n_{s} \times 3}$ and $\boldsymbol{\beta}_{s}=\left[\beta_{1}, \beta_{2}, \ldots, \beta_{n_{s}}\right]^{T} \in \mathbb{R}^{n_{s}}$ is the vector of the angles of the steering wheels of the robot. The mobility of a robot is directly related to the rank of $\mathbf{C}_{1}^{*}\left(\boldsymbol{\beta}_{s}\right)$ which depends on the robot design [21]. The degree of mobility $\delta_{m}$ of a robot is defined as:

$$
\delta_{m}=\operatorname{nul}\left(\mathbf{C}_{1}^{*}\left(\boldsymbol{\beta}_{s}\right)\right)=3-\operatorname{rank}\left(\mathbf{C}_{1}^{*}\left(\boldsymbol{\beta}_{s}\right)\right),
$$

which is equal to the number of degrees of freedom that can be directly manipulated from the robot's inputs without reorientation of the steering wheels. On the other hand, the degree of steerability $\delta_{s}$ of a robot is defined as:

$$
\delta_{s}=\operatorname{rank}\left(\mathbf{C}_{1 s}\left(\boldsymbol{\beta}_{s}\right)\right)
$$

which corresponds to the number of steering wheels that can be oriented independently in order to steer the robot. If a robot is equipped with more than $\delta_{s}$ steering wheels, the motion of the extra ones must be coordinated in order to guarantee the existence of the Instantaneous Center of Rotation (ICR) at all times [22]. Finally, the degree of maneuverability $\delta_{M}$ of a robot is defined as $\delta_{M}=\delta_{m}+\delta_{s}$.

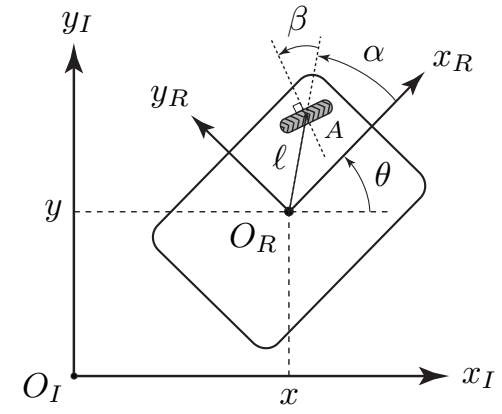

Fig. 1. Notation for a conventional centered wheel of a mobile robot.

For WMRs of practical interest, the indices $\delta_{m}$ and $\delta_{s}$ must comply with a set of constraints [21]. Therefore, only four structures are relevant in practice, corresponding to the following four pairs:

$$
\begin{array}{c|cccc}
\delta_{m} & 2 & 2 & 1 & 1 \\
\delta_{s} & 0 & 1 & 1 & 2
\end{array}
$$

Each structure is referred to as a type $\left(\delta_{m}, \delta_{s}\right)$ robot. Note that in $(2)$, we omitted type $(3,0)$ robots, since they do not have fixed or centered steering wheels (in fact, "omnimobiles", which have full mobility in the plane, are only equipped with Swedish or off-centered orientable wheels).

Remark 1: Type $(2,0)$ robots have no steering wheels, but either one or several fixed wheels with a common axle: a typical example is the unicycle robot [21]. Type $(2,1)$ robots have no fixed wheels and at least one steering wheel: if there is more than one steering wheel, their orientation must be coordinated in such a way that $\delta_{s}=1$. Type $(1,1)$ robots have one or several fixed wheels on a single common axle, and also one or several steering wheels, under the condition that their centers are not located on the common axle of the fixed wheels and that their orientation is coordinated. Car-like robots belong to this class. Finally, type $(1,2)$ robots have no fixed wheels, but at least two steering wheels: if there are more than two steering wheels, then their orientation must be coordinated in order to satisfy the condition $\delta_{s}=2$. A typical example is the two-steer robot [23, Sect. 3.3].

\section{MOBILITY OF DISTANCE-BEARING FORMATIONS OF UNICYCLE ROBOTS}

In this section, we extend the classification by type presented in Sect. II, to formations of unicycle robots defined by relative distance and bearing constraints. A generic $n$-robot formation can be encoded in terms of the weighted undirected graph $\mathcal{G}_{d}=\left(V, E_{d}, \mathbf{D}\right)$ and the weighted directed graph $\mathcal{G}_{\phi}=\left(V, E_{\phi}, \boldsymbol{\Phi}\right)$, whose set of vertices $V=$ $\{1,2, \ldots, n\}$ is indexed by the robots in the team, and whose set of edges $E_{d}\left(E_{\phi}\right)$ contains unordered (ordered) pairs of vertices that represent inter-robot formation specifications. $\mathbf{D}=\left[d_{i j}\right], \boldsymbol{\Phi}=\left[\phi_{i j}\right]$ are the $n \times n$ weighted adjacency matrices of distance and bearing constraints, respectively, where,

$\left\{\begin{array}{ll}d_{i j}>0, & \text { if }\{i, j\} \in E_{d}, \\ d_{i j}=0, & \text { otherwise, }\end{array} \quad \begin{cases}\phi_{i j}>0, & \text { if }(i, j) \in E_{\phi}, \\ \phi_{i j}=0, & \text { otherwise. }\end{cases}\right.$ 


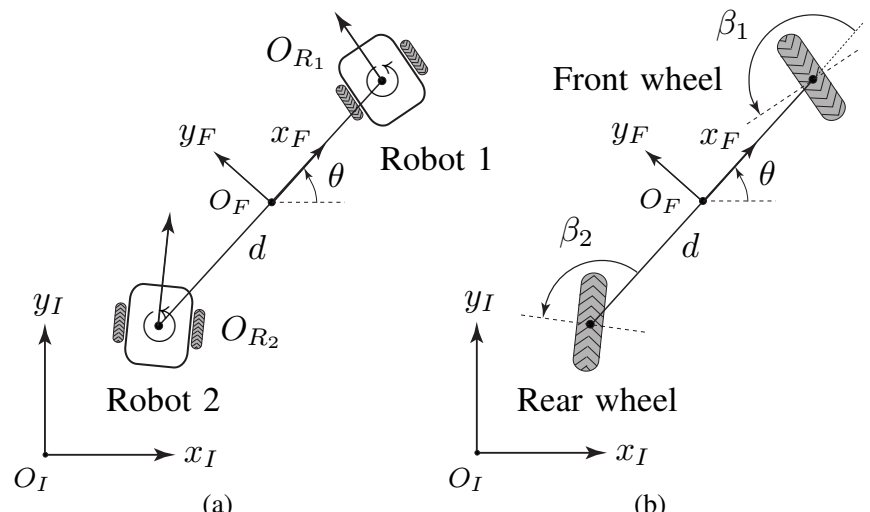

(a)

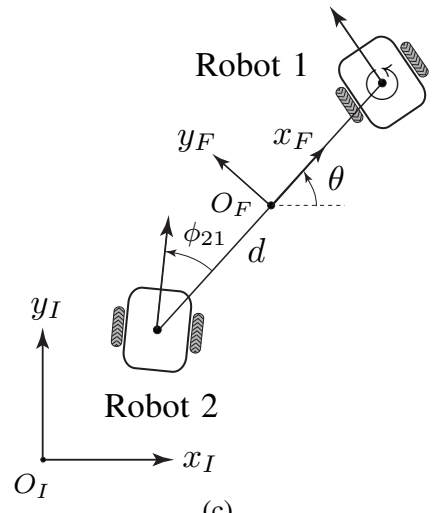

(c)

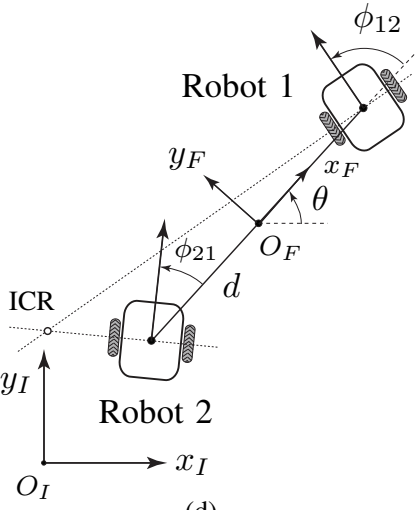

(d)

Fig. 2. Two unicycles. (a) Formation defined by a single distance constraint, $d$, and (b) corresponding m-robot after the application of the reduction procedure. The formation in (c) is defined by a distance constraint, $d$, and a bearing constraint, $\phi_{21}$. The formation in (d) is defined by a distance constraint, $d$, and two bearing constraints, $\phi_{12}, \phi_{21}$ with $\phi_{12} \neq \phi_{21}$. The symbol $\circlearrowleft$ indicates that a robot can freely rotate about its vertical axis.

For the sake of exposition, sacrificing generality for conceptual simplicity and insight, in the rest of this paper we will make the following assumption:

Assumption 1: The $n \geq 2$ robots are disposed at the vertices of a regular convex polygon of side $d$, and the origin $O_{F}$ of the reference frame $\left\{O_{F} ; x_{F}, y_{F}\right\}$ of the formation is placed at the circumcenter of the polygon, with the $x_{F}$-axis pointing towards robot 1 .

Note that while the last choice is arbitrary, it will allow us to easily draw a parallel between the single-robot case studied in Sect. II and the multi-robot case, via the notion of "macro-robot". We also observe that, by definition, the formations studied in this paper are rigid [8]. In what follows, we shall proceed in stages and analyze formations with an increasing number of robots. We will use the terms "m-robot" and "m-wheel" as shorthands for macro-robot and for a macro-robot's wheel, respectively.

\section{A. Formation of two unicycles}

Fig. 2(a) shows a formation of two unicycle robots defined by a single distance constraint (the distance $d$ between their centers $O_{R_{1}}$ and $O_{R_{2}}$ ). In this case, $V=\{1,2\}, E_{d}=$ $\{\{1,2\}\}, E_{\phi}=\emptyset$ (the empty set), and $n_{f}+n_{s}=0+2$. The mobility analysis for this formation reduces to that of the m-robot reported in Fig. 2(b), whose m-wheels are separated by the distance $d$. By denoting the orientation of the front (rear) steering $\mathrm{m}$-wheel with respect to the "chassis" of the m-robot by $\beta_{1}\left(\beta_{2}\right)$, and by noticing that for the front $\mathrm{m}$-wheel $\alpha_{1}=0$ and for the rear m-wheel $\alpha_{2}=\pi$ (cf. Fig. 1), we have that:

$$
\mathbf{C}_{1}^{*}\left(\beta_{1}, \beta_{2}\right)=\left[\begin{array}{ccc}
\cos \beta_{1} & \sin \beta_{1} & \frac{1}{2} d \sin \beta_{1} \\
-\cos \beta_{2} & -\sin \beta_{2} & \frac{1}{2} d \sin \beta_{2}
\end{array}\right] .
$$

Therefore, $\left(\delta_{m}, \delta_{s}\right)=(1,2), \delta_{M}=3$, and the formation behaves as a two-steer robot.

Fig. 2(c) reports a formation of two unicycles defined by a distance and a bearing constraint. On par with the previous case, $V=\{1,2\}$ and $E_{d}=\{\{1,2\}\}$, but now $E_{\phi}=\{(2,1)\}$. By applying the same formation reduction procedure as above, we have that the front m-wheel of the associated $\mathrm{m}$-robot is steerable with an angle $\beta_{1}$ and that $\alpha_{1}=0$. On the other hand, the rear m-wheel of the m-robot is now fixed with constant angle $\beta_{2}=\phi_{21}+\pi / 2$ and $\alpha_{2}=\pi$. This entails that $\left(n_{f}+n_{s}=1+1\right)$ :

$$
\mathbf{C}_{1}^{*}\left(\beta_{1}\right)=\left[\begin{array}{ccc}
\sin \phi_{21} & -\cos \phi_{21} & \frac{1}{2} d \cos \phi_{21} \\
\cos \beta_{1} & \sin \beta_{1} & \frac{1}{2} d \sin \beta_{1}
\end{array}\right],
$$

and thus $\left(\delta_{m}, \delta_{s}\right)=(1,1)$, meaning that the formation is equivalent to a car-like robot.

Finally, Fig. 2(d) shows the case of $E_{d}=\{\{1,2\}\}$ and $E_{\phi}=\{(1,2),(2,1)\}$. The m-wheels of the corresponding m-robot are both fixed, thus $\delta_{s}=0$. Note that if $\phi_{12} \neq \phi_{21}$, this formation is not of practical interest, since the only possible motion is about the fixed ICR of the m-robot. However, if $\phi_{12}=\phi_{21} \neq 0$, the m-wheels of the m-robot are parallel and $\left(\delta_{m}, \delta_{s}\right)=(2,0)$, i.e. the formation behaves as a unicycle robot.

\section{B. Formation of three unicycles}

Fig. 3(a) shows a formation defined by three distance constraints: $V=\{1,2,3\}$ and $E_{d}=\{\{1,2\},\{2,3\},\{3,1\}\}$. The reduction procedure described in Sect. III-A can be applied to this formation as well. By noticing that $\alpha_{1}=0$, $\alpha_{2}=2 \pi / 3, \alpha_{3}=-2 \pi / 3$, and that the circumradius of the equilateral triangle in Fig. 3(a) is $\sqrt{3} d / 3$, we obtain:

$$
\mathbf{C}_{1}^{*}\left(\beta_{1}, \beta_{2}, \beta_{3}\right)=\left[\begin{array}{ccc}
\cos \beta_{1} & \sin \beta_{1} & \frac{\sqrt{3}}{3} d \sin \beta_{1} \\
\cos \left(\beta_{2}+\frac{2 \pi}{3}\right) & \sin \left(\beta_{2}+\frac{2 \pi}{3}\right) & \frac{\sqrt{3}}{3} d \sin \beta_{2} \\
\cos \left(\beta_{3}-\frac{2 \pi}{3}\right) & \sin \left(\beta_{3}-\frac{2 \pi}{3}\right) & \frac{\sqrt{3}}{3} d \sin \beta_{3}
\end{array}\right] .
$$

In order to gain some insight into the mobility properties of the formation, let us study the determinant of $\mathbf{C}_{1}^{*}\left(\beta_{1}, \beta_{2}, \beta_{3}\right)$. By elementary trigonometry, we find that:

$$
\begin{aligned}
& \operatorname{det}\left(\mathbf{C}_{1}^{*}\left(\beta_{1}, \beta_{2}, \beta_{3}\right)\right)=\frac{d}{2}\left[\sin \left(-\beta_{1}+\beta_{2}+\beta_{3}\right)+\right. \\
&\left.\sin \left(\beta_{1}-\beta_{2}+\beta_{3}\right)+\sin \left(\beta_{1}+\beta_{2}-\beta_{3}\right)\right] .
\end{aligned}
$$

Although it is not trivial to provide an exhaustive characterization of the zeros of this transcendental function of $\beta_{1}, \beta_{2}$ and $\beta_{3}$, we can easily identify the following special configurations of the m-wheels:

1) Let $\beta_{1}=\beta_{1}(t)$ (free), $\beta_{2}=\beta_{1} \pm \pi / 3, \beta_{3}=\beta_{1} \mp \pi / 3$. The orientation of the three m-wheels of the m-robot 


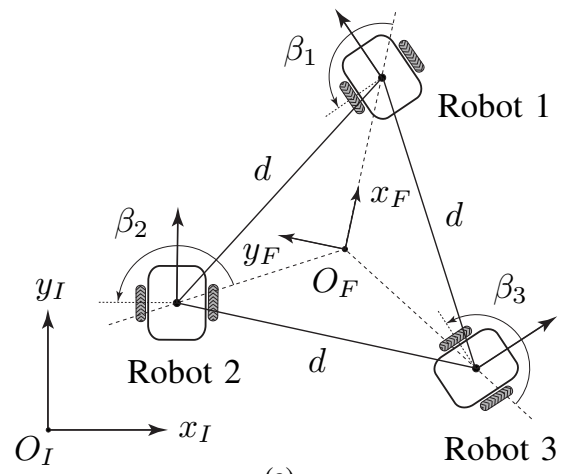

(a)

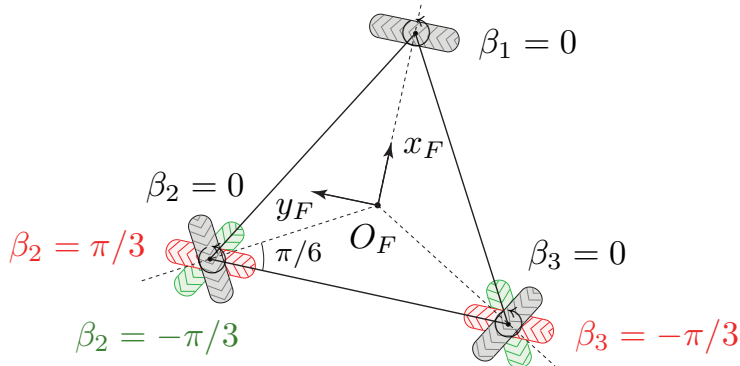

(b)

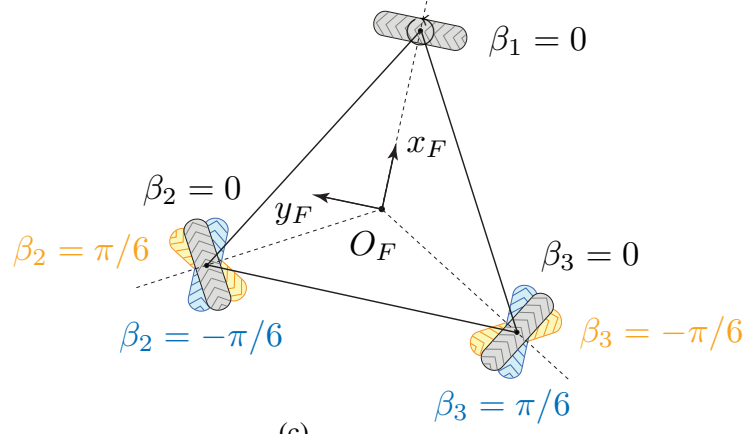

(c)

Fig. 3. Three unicycles. (a) Formation defined by three distance constraints; (b), (c) Special orientations of the m-wheels of the m-robot (for the sake of illustration, we set $\beta_{1}=0$ in both cases).

is coupled (red and green, respectively, in Fig. 3(b)), and the formation behaves as a three-wheel synchrodrive robot [22]. It is well-known that the kinematic model of a synchro-drive robot is equivalent to that of a type $(1,1)$ robot [21], [23, Sect. 3.3.3]. In fact, a synchro-drive robot can only manipulate, in total, two degrees of freedom (the orientation of the chassis cannot be changed).

2) Let $\beta_{1}=\beta_{1}(t)$ (free), $\beta_{2}=-\pi / 6, \beta_{3}=\pi / 6$ (cyan in Fig. 3(c)). The m-wheels 2 and 3 are fixed and parallel to the $x_{F}$-axis, hence the formation type is $(1,1)$.

3) Let $\beta_{1}=\beta_{1}(t)$ (free), $\beta_{2}=\pi / 6$ and $\beta_{3}=-\pi / 6$ (yellow in Fig. 3(c)). The axles of m-wheels 2 and 3 concur at the center of m-wheel 1 (the ICR), and the only possible motion of the formation is about this fixed point. This arrangement is called singular wheels configuration (SWC) in [19, Def. 2].

4) Let $\beta_{1}=\beta_{2}=\beta_{3}=0$. The three m-wheels are tangent to the circle circumscribing the triangular "chassis" of the m-robot (gray in Fig. 3(b) or Fig. 3(c)). In this degenerate configuration, the only possible motion is a rotation about the center $O_{F}$ (the ICR) of the formation.

\section{Formation of $n$ unicycles}

Fig. 4 reports a formation of $n \geq 3$ unicycles defined by $n$ distance constraints: $V=\{1,2, \ldots, n\}$ and $E_{d}=$ $\{\{1,2\},\{2,3\}, \ldots,\{n, 1\}\}$. Recall that the central angle of a regular convex polygon with $n$ vertices is $\gamma=2 \pi / n$ and that the circumradius is $r_{c}=d /(2 \sin (\pi / n))$. Hence, $\alpha_{i}=\gamma(i-1), i \in\{1,2, \ldots, n\}$. By applying the reduction procedure seen in the previous sections, we obtain:

$$
\begin{aligned}
& \mathbf{C}_{1}^{*}\left(\beta_{1}, \ldots, \beta_{n}\right)= \\
& {\left[\begin{array}{ccc}
\cos \beta_{1} & \sin \beta_{1} & r_{c} \sin \beta_{1} \\
\cos \left(\beta_{2}+\gamma\right) & \sin \left(\beta_{2}+\gamma\right) & r_{c} \sin \beta_{2} \\
\vdots & \vdots & \vdots \\
\cos \left(\beta_{n}+\gamma(n-1)\right) & \sin \left(\beta_{n}+\gamma(n-1)\right) & r_{c} \sin \beta_{n}
\end{array}\right] .}
\end{aligned}
$$

The next result is instrumental in studying the rank deficiency of $\mathbf{C}_{1}^{*}\left(\beta_{1}, \ldots, \beta_{n}\right) \in \mathbb{R}^{n \times 3}$.

Proposition 1: The $n+1$ points $O_{R_{1}}, O_{R_{2}}, \ldots, O_{R_{n}}, O_{F}$ obey the classic Descartes' principle of instantaneous motion, i.e., at each instant, their motion coincides either with a pure translation or with a pure rotation about the ICR.

Proof: The distance between any two given points in $\left\{O_{R_{1}}, O_{R_{2}}, \ldots, O_{R_{n}}, O_{F}\right\}$ remains constant over time in the horizontal plane defined by $\left\{O_{I} ; x_{I}, y_{I}\right\}$. Hence, the proof is the same as that traditionally used for a rigid body in mechanics.

Lemma 1: The following condition holds true:

$$
1<\operatorname{rank}\left(\mathbf{C}_{1}^{*}\left(\beta_{1}, \ldots, \beta_{n}\right)\right) \leq 3 .
$$

Proof: Let $\mathbf{c}=\left[c_{i}\right] \in \mathbb{R}^{n}$ with $c_{i}=\cos \left(\beta_{i}+\gamma(i-1)\right)$, $\mathbf{s}=\left[s_{i}\right] \in \mathbb{R}^{n}$ with $s_{i}=\sin \left(\beta_{i}+\gamma(i-1)\right)$, and $\mathbf{t}=\left[t_{i}\right] \in \mathbb{R}^{n}$ with $t_{i}=r_{c} \sin \beta_{i}, i \in\{1,2, \ldots, n\}$, then $\operatorname{rank}\left(\mathbf{C}_{1}^{*}\left(\beta_{1}, \ldots, \beta_{n}\right)\right)=\operatorname{rank}([\mathbf{c}, \mathbf{s}, \mathbf{t}]) \leq 3$. Moreover, we have that $\operatorname{rank}\left(\mathbf{C}_{1}^{*}\left(\beta_{1}, \ldots, \beta_{n}\right)\right) \geq 1$, since, trivially, $\operatorname{rank}([\mathbf{c}, \mathbf{s}]) \geq 1$. The inequality $\operatorname{rank}([\mathbf{c}, \mathbf{s}]) \leq$

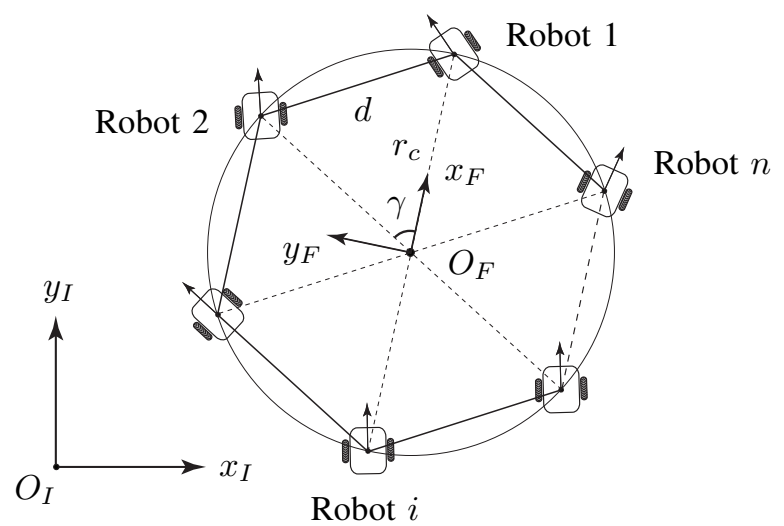

Fig. 4. $n$ unicycles. The formation is specified by $n$ distance constraints. The regular convex polygon has side $d$ and circumradius $r_{c}$. 
$\operatorname{rank}([\mathbf{c}, \mathbf{s}, \mathbf{t}])$ gives us $\operatorname{rank}([\mathbf{c}, \mathbf{s}])=1$ as a necessary condition for $\operatorname{rank}\left(\mathbf{C}_{1}^{*}\left(\beta_{1}, \ldots, \beta_{n}\right)\right)=1$. Three cases correspond to $\operatorname{rank}([\mathbf{c}, \mathbf{s}])=1$ :

1) $\mathbf{c}=\mathbf{0} \Leftrightarrow \beta_{i}+\gamma(i-1)=(2 h+1) \pi / 2, h \in \mathbb{Z}, \forall i$. But then $s_{i}= \pm 1, t_{i}= \pm r_{c} \cos \gamma(i-1)$, and thus $\operatorname{rank}([\mathbf{s}, \mathbf{t}])=2$. Hence, this case can be ruled out.

2) $\mathbf{s}=\mathbf{0} \Leftrightarrow \beta_{i}+\gamma(i-1)=h \pi, h \in \mathbb{Z}, \forall i$. But then $c_{i}= \pm 1, t_{i}=\mp r_{c} \sin \gamma(i-1)$, and $\operatorname{rank}([\mathbf{c}, \mathbf{t}])=2$. Therefore, this case should be excluded too.

3) $\mathbf{s}=\lambda \mathbf{c}$ with $\lambda \in \mathbb{R} \backslash\{0\} \Leftrightarrow \mathbf{c} \neq \mathbf{0}$ and $s_{i}=\lambda c_{i}, \forall i$ (in fact, the sine and cosine of the same angle cannot simultaneously be zero). Therefore, $c_{i} \neq 0, s_{i} \neq 0$ and $\tan \left(\beta_{i}+\gamma(i-1)\right)=\lambda$, $\forall i$. The angle $\vartheta=\arctan (\lambda)$, then verifies: $\vartheta \neq$ $h \pi / 2$ and $\beta_{i}+\gamma(i-1)=\vartheta+k_{i} \pi, h, k_{i} \in \mathbb{Z}$. Hence, $\operatorname{rank}\left(\mathbf{C}_{1}^{*}\left(\beta_{1}, \ldots, \beta_{n}\right)\right)=\operatorname{rank}([\mathbf{c}, \mathbf{t}])$ with $c_{i}=$ $\cos \left(\vartheta+k_{i} \pi\right)=(-1)^{k_{i}} \cos \vartheta$ and $t_{i}=r_{c} \sin (\vartheta+$ $\left.k_{i} \pi-\gamma(i-1)\right)=(-1)^{k_{i}} r_{c} \sin (\vartheta-\gamma(i-1))$. Then, a necessary condition for $\operatorname{rank}\left(\mathbf{C}_{1}^{*}\left(\beta_{1}, \ldots, \beta_{n}\right)\right)=1$ is $\mathbf{t}=\mathbf{0}$ or $\mathbf{t}=\rho \mathbf{c}$ with $\rho \in \mathbb{R} \backslash\{0\} . \mathbf{t}=\mathbf{0}$ should be ruled out, since for $i=1, t_{1}=(-1)^{k_{1}} r_{c} \sin \vartheta \neq 0$. Let us now suppose that $\mathbf{t}=\rho \mathbf{c}$. Then, $\rho=r_{c} \tan \vartheta$ $(i=1)$ and therefore $\sin (\vartheta-\gamma)=\sin \vartheta$ with $\gamma=2 \pi / n<2 \pi / 3(i=2)$. Therefore, also this case is not acceptable.

In conclusion, condition (3) holds true.

The following lemma provides an algebraic characterization of the full-rank case in Lemma 1.

Lemma 2: $\operatorname{rank}\left(\mathbf{C}_{1}^{*}\left(\beta_{1}, \ldots, \beta_{n}\right)\right)=3$ if and only if there exist $i, j, k \in\{1,2, \ldots, n\}, i \neq j \neq k$, such that:

$\sin \beta_{i} \sin \left(\beta_{k}-\beta_{j}+\gamma(k-j)\right)-\sin \beta_{j} \sin \left(\beta_{k}-\beta_{i}+\gamma(k-i)\right)$

$+\sin \beta_{k} \sin \left(\beta_{j}-\beta_{i}+\gamma(j-i)\right) \neq 0$.

Proof: In view of [24, Prop. 2.7.7], the rank of $\mathbf{C}_{1}^{*}\left(\beta_{1}, \ldots, \beta_{n}\right)$ is the largest order of all nonsingular submatrices of $\mathbf{C}_{1}^{*}\left(\beta_{1}, \ldots, \beta_{n}\right)$ (or equivalently, the maximal order of a non-zero minor of $\mathbf{C}_{1}^{*}\left(\beta_{1}, \ldots, \beta_{n}\right)$ ). Let us consider the generic submatrix $\overline{\mathbf{C}}_{1}^{*}\left(\beta_{i}, \beta_{j}, \beta_{k}\right) \in \mathbb{R}^{3 \times 3}$ composed of the $i$ th, $j$ th and $k$ th row of $\mathbf{C}_{1}^{*}\left(\beta_{1}, \ldots, \beta_{n}\right)$, with $i<j<k$, for the sake of simplicity:

$$
\begin{aligned}
& \overline{\mathbf{C}}_{1}^{*}\left(\beta_{i}, \beta_{j}, \beta_{k}\right)= \\
& {\left[\begin{array}{ccc}
\cos \left(\beta_{i}+\gamma(i-1)\right) & \sin \left(\beta_{i}+\gamma(i-1)\right) & r_{c} \sin \beta_{i} \\
\cos \left(\beta_{j}+\gamma(j-1)\right) & \sin \left(\beta_{j}+\gamma(j-1)\right) & r_{c} \sin \beta_{j} \\
\cos \left(\beta_{k}+\gamma(k-1)\right) & \sin \left(\beta_{k}+\gamma(k-1)\right) & r_{c} \sin \beta_{k}
\end{array}\right] .}
\end{aligned}
$$

If $\operatorname{det}\left(\overline{\mathbf{C}}_{1}^{*}\left(\beta_{i}, \beta_{j}, \beta_{k}\right)\right) \neq 0$, then $\mathbf{C}_{1}^{*}\left(\beta_{1}, \ldots, \beta_{n}\right)$ has full rank. By using the Laplace expansion along the third column of $\overline{\mathbf{C}}_{1}^{*}\left(\beta_{i}, \beta_{j}, \beta_{k}\right)$ and the angle addition formula for sine, we end up with $\operatorname{det}\left(\overline{\mathbf{C}}_{1}^{*}\left(\beta_{i}, \beta_{j}, \beta_{k}\right)\right)=r_{c}\left[\sin \beta_{i} \sin \left(\beta_{k}-\beta_{j}+\right.\right.$ $\gamma(k-j))-\sin \beta_{j} \sin \left(\beta_{k}-\beta_{i}+\gamma(k-i)\right)+\sin \beta_{k} \sin \left(\beta_{j}-\right.$ $\left.\left.\beta_{i}+\gamma(j-i)\right)\right]$ which yields condition (4).

In the next proposition, we use geometric arguments to show that, actually, only two types are admissible for nontrivial $n$-robot formations.

Proposition 2: Under the previous assumptions, a formation of $n \geq 3$ unicycles defined by $n$ distance constraints is either of type $(1,1)$ or $(1,2)$.
Proof: For the sake of simplicity, let us suppose that $i<j<k$ (cf. Lemma 2). Let $L_{i}, i \in\{1,2, \ldots, n\}$, be the straight line passing through $O_{R_{i}}$ and parallel to the axle of the wheels of robot $i$. With respect to $\left\{O_{F} ; x_{F}, y_{F}\right\}$, the straight line $L_{i}, i \in\{1,2, \ldots, n\}$, passes through $O_{R_{i}}=$ $\left(r_{c} \cos (\gamma(i-1)), r_{c} \sin (\gamma(i-1))\right)$, and it has an angle of incline $\beta_{i}+\gamma(i-1)$. If $\beta_{i}+\gamma(i-1) \neq(2 h+1) \pi / 2, h \in \mathbb{Z}$, this line can be expressed by,

$$
\begin{aligned}
y= & \tan \left(\beta_{i}+\gamma(i-1)\right) x+r_{c}[\sin (\gamma(i-1)) \\
& \left.-\cos (\gamma(i-1)) \tan \left(\beta_{i}+\gamma(i-1)\right)\right],
\end{aligned}
$$

where $x$ is the independent variable. By multiplying both sides of (5) by $\cos \left(\beta_{i}+\gamma(i-1)\right)$, we obtain $a_{i} x+$ $b_{i} y+c_{i}=0$ where $a_{i}=-\sin \left(\beta_{i}+\gamma(i-1)\right), b_{i}=$ $\cos \left(\beta_{i}+\gamma(i-1)\right)$ and $c_{i}=-r_{c} \sin \beta_{i}$. If $\beta_{i}+\gamma(i-1)=$ $(2 h+1) \pi / 2, h \in \mathbb{Z}$, line $L_{i}$ can be expressed by $a_{i} x+c_{i}=0$ where $a_{i}=(-1)^{h} \neq 0, b_{i}=0$ and $c_{i}=(-1)^{h} r_{c} \cos (\gamma(i-$ $1)$ ). By definition, $L_{i}$ is always orthogonal at $O_{R_{i}}$ to the velocity vector of $O_{R_{i}}$ in $\left\{O_{I} ; x_{I}, y_{I}\right\}$. Moreover $\left(a_{i}, b_{i}\right) \neq$ $(0,0), \forall i$. Owing to Prop. 1 , the points $\left\{O_{R_{i}}, O_{R_{j}}, O_{R_{k}}\right\}$ obey the classic Descartes' principle of instantaneous motion: i.e. at each instant, their motion coincides either with a pure translation $\left(L_{i}, L_{j}\right.$ and $L_{k}$ are parallel) or with a pure rotation about the $\operatorname{ICR}\left(L_{i}, L_{j}\right.$ and $L_{k}$ concur at the ICR location). According to [25, p. 41], three straight lines $L_{i}$, $L_{j}$ and $L_{k}$ intersect at a point or are parallel if and only if $\operatorname{det}\left(\mathbf{B}\left(\beta_{i}, \beta_{j}, \beta_{k}\right)\right)=0$, where $\mathbf{B}\left(\beta_{i}, \beta_{j}, \beta_{k}\right)=\left[\begin{array}{lll}a_{i} & b_{i} & c_{i} \\ a_{j} & b_{j} & c_{j} \\ a_{k} & b_{k} & c_{k}\end{array}\right]$. Recall that if the row of a square matrix is multiplied by -1 or if any pair of its rows is interchanged, then its determinant is multiplied by -1 . Since we have three sign changes, then $\operatorname{det}\left(\overline{\mathbf{C}}_{1}^{*}\left(\beta_{i}, \beta_{j}, \beta_{k}\right)\right)=-\operatorname{det}\left(\mathbf{B}\left(\beta_{i}, \beta_{j}, \beta_{k}\right)\right)$. By virtue of Lemma $2, \mathbf{C}_{1}^{*}\left(\beta_{1}, \ldots, \beta_{n}\right)$ has full rank if and only if there exist three different integers $i, j, k \in\{1,2, \ldots, n\}$, such that $\operatorname{det}\left(\overline{\mathbf{C}}_{1}^{*}\left(\beta_{i}, \beta_{j}, \beta_{k}\right)\right) \neq 0$. But because of Prop. 1, this condition does not hold true, from which, owing to (2), the statement directly follows.

To make the link with the special configurations studied in Sect. III-B, we list below their $n$-robot counterparts.

1) Let $\beta_{1}=\beta_{1}(t)$ (free), $\beta_{k}=\beta_{1}+2 \gamma(k-1)$, $k \in\{2,3, \ldots, n\}$. The orientation of the $n$ m-wheels is coupled and the formation behaves as an $n$-wheel synchro-drive robot (cf. Case 1 in Sect. III-B).

2) Let $\beta_{1}=\beta_{1}(t)$ (free), $\beta_{k}=\beta_{1}-\gamma(k-1), k \in$ $\{2,3, \ldots, n\}$. As in the previous case, the orientation of the $n$ m-wheels of the m-robot is coupled (cf. Case 1 and 2 in Sect. III-B).

3) For $n$ even, let $\beta_{1}=\beta_{1}(t)$ (free), $\beta_{2}=\gamma(n-2) / 4$, $\ldots, \beta_{(n / 2)-1}=\gamma, \beta_{n / 2}=\gamma / 2, \beta_{(n / 2)+1}=\pi$, $\beta_{(n / 2)+2}=\pi-\gamma / 2, \beta_{(n / 2)+3}=\pi-\gamma, \ldots, \beta_{n}=\pi-$ $\gamma(n-2) / 4$, and for $n$ odd, let $\beta_{1}=\beta_{1}(t)$ (free), $\beta_{2}=$ $\gamma(n-2) / 4, \ldots, \beta_{(n+1) / 2-2}=5 \gamma / 4, \beta_{(n+1) / 2-1}=$ $3 \gamma / 4, \beta_{(n+1) / 2}=\gamma / 4, \beta_{(n+1) / 2+1}=\pi-\gamma / 4$, $\beta_{(n+1) / 2+2}=\pi-3 \gamma / 4, \ldots, \beta_{n}=\pi-\gamma(n-2) / 4$. The axles of $\mathrm{m}$-wheels $2,3, \ldots, n$ concur at the center of m-wheel 1 (the ICR), and the formation can only rotate about this fixed point (cf. Case 3 in Sect. III-B).

4) Let $\beta_{1}=\beta_{2}=\ldots=\beta_{n}=0$. The m-wheels 
are tangent to the circle circumscribing the polygonal "chassis" of the m-robot. The only possible motion is a rotation about the center $O_{F}$ of the formation (cf. Case 4 in Sect. III-B).

Remark 2 (Reconfigurable formations): With reference to Fig. 1, if the polar coordinates $(\ell, \alpha)$ used to express the position of the center $A$ of the wheel with respect to $O_{R}$ are time-varying, condition (1) becomes:

$$
\begin{aligned}
& {[\cos (\alpha+\beta), \sin (\alpha+\beta), \ell \sin \beta] \mathbf{R}^{T}(\theta) \dot{\mathbf{q}}+} \\
& \quad[\cos \beta, \ell \sin \beta] \dot{\boldsymbol{\tau}}=0,
\end{aligned}
$$

where $\tau=[\ell, \alpha]^{T}$. Eq. (6) can be obtained by plugging the coordinates of point $A,\left(x_{A}, y_{A}\right)=\left(x+\ell \cos (\theta+\alpha), y_{A}=\right.$ $y+\ell \sin (\theta+\alpha))$, into the nonslip condition $\dot{y}_{A} \cos (\theta+\alpha+$ $\beta+\pi / 2)-\dot{x}_{A} \sin (\theta+\alpha+\beta+\pi / 2)=0$. The generalized nonslip condition (6) can be used to study the mobility of reconfigurable formations, i.e. formations whose shape can vary over time. While formations able to contract/expand are relevant in the applications, the extension of our taxonomy to these structures goes beyond the scope of this paper, and it is reserved as future work.

\section{TRAJECTORY-TRACKING CONTROL OF A FORMATION OF UNICYCLES}

In this section, we adapt the trajectory-tracking control law via dynamic feedback originally introduced in [19, Sect. III] for mobile robots equipped with multiple steering wheels, to distance-bearing formations of unicycles. This task is made easy by the proposed notion of macro-robot. Note that we are not interested here in a stabilization problem, i.e. we assume that the unicycles satisfy the formation constraints initially, and such constraints should be respected at all times.

Following [19], let us describe the position of the ICR of the formation using the polar coordinates $(1 / \sigma, \zeta)$ expressed in the frame centered at $\mathrm{m}$-wheel 1 (whose axes are parallel to the longitudinal and lateral axes of the "chassis" of the m-robot, respectively). In particular, $1 / \sigma$ is the (signed) instantaneous radius of curvature of the m-robot viewed from the frame of m-wheel 1 and $\zeta$ is the angle between the $x$-axis of the reference frame of $\mathrm{m}$-wheel 1 and its axle $\mathbf{r}=[\cos \zeta, \sin \zeta]^{T}$, namely,

$$
\sigma= \begin{cases}0, & \text { if the ICR is at infinity, } \\ \frac{\operatorname{sgn}\left(\left\langle\mathbf{r}, \mathbf{w}_{1, \mathrm{ICR}}\right\rangle\right)}{\left\|\mathbf{w}_{1, \mathrm{ICR}}\right\|}, & \text { otherwise, }\end{cases}
$$

where $\mathbf{w}_{1, \text { ICR }}$ is the vector from the center of $\mathrm{m}$-wheel 1 to the ICR, and $\operatorname{sgn}(\cdot)$ denotes the sign function (see Fig. 4 in [19]). Let us introduce the state vector $\mathbf{x}=$ $[x, y, \theta, \zeta, \sigma]^{T}$ and the input vector $\mathbf{u}=\left[\eta_{1}, \eta_{2}, \eta_{3}\right]^{T}$, where $[x, y, \theta]^{T}$ now denotes the pose of the frame of m-wheel 1 with respect to $\left\{O_{I} ; x_{I}, y_{I}\right\}, \eta_{1}$ is the linear velocity of m-wheel $1, \eta_{2}=\dot{\zeta}$ and $\eta_{3}=\dot{\sigma}$. We can then write the following state-space model for the m-robot:

$$
\dot{\mathbf{x}}=\left[\begin{array}{ccc}
\sin (\theta+\zeta) & 0 & 0 \\
-\cos (\theta+\zeta) & 0 & 0 \\
\sigma & 0 & 0 \\
0 & 1 & 0 \\
0 & 0 & 1
\end{array}\right] \mathbf{u}
$$

Note that this model is completely independent of the number of m-wheels and of their disposition in the "chassis" of the m-robot. As shown in [19, Th. 1], system (7) can be fully dynamically linearized if SWCs (system's intrinsic singularities) and rest configurations (singularities of the linearizing control law) are not met. A candidate output vector which provides such a linearization is $\varphi=\left[x, y, \theta / \sigma_{F}\right]^{T}$ where $\sigma_{F} \triangleq \min \left\{\sigma_{2}, \sigma_{3}, \ldots, \sigma_{n}\right\}$, being $\left(1 / \sigma_{j}, \zeta_{j}\right), j \in$ $\{2,3, \ldots, n\}$, the polar coordinates of $\mathrm{m}$-wheel $j$ expressed in the frame of m-wheel 1 . Note that $\sigma_{F}>0$ is a characteristic constant of the formation considered. Using the dynamic extension algorithm, the input $\eta_{1}$ can be delayed. This leads to the following extended model for the m-robot (cf. eq. (7)),

$$
\dot{\mathbf{x}}_{e}=\left[\begin{array}{c}
\eta_{1} \sin (\theta+\zeta) \\
-\eta_{1} \cos (\theta+\zeta) \\
\sigma \eta_{1} / \sigma_{F} \\
\mathbf{0}_{3 \times 1}
\end{array}\right]+\left[\begin{array}{c}
\mathbf{0}_{3 \times 3} \\
\mathbf{I}_{3 \times 3}
\end{array}\right] \mathbf{u}_{e}
$$

where $\mathbf{x}_{e}=\left[x, y, \theta / \sigma_{F}, \zeta, \sigma, \eta_{1}\right]^{T}$ and $\mathbf{u}_{e}=\left[\eta_{2}, \eta_{3}, \eta_{4}\right]^{T}$ are the extended state and input vectors, respectively. Let now $\varphi_{\text {ref }}(t)=\left[x_{\text {ref }}(t), y_{\text {ref }}(t), \theta_{\text {ref }}(t) / \sigma_{F}\right]^{T} \in \mathcal{C}^{2}$ be the reference trajectory that the output vector $\varphi(t)$ should follow, and let $\mathbf{H}_{1}, \mathbf{H}_{2} \in \mathbb{R}^{3 \times 3}$ be two diagonal positive-definite gain matrices. Then, as long as $\eta_{1} \neq 0$ (i.e. with the proviso that singular rest configurations are avoided), trajectory tracking for system (8) is achieved via:

$$
\begin{gathered}
\mathbf{u}_{e}=\boldsymbol{\Delta}^{-1}\left(\mathbf{x}_{e}\right)\left(\mathbf{u}_{a}-\left[\begin{array}{c}
\sigma \eta_{1}^{2} \cos (\theta+\zeta) \\
\sigma \eta_{1}^{2} \sin (\theta+\zeta) \\
0
\end{array}\right]\right), \\
\boldsymbol{\Delta}\left(\mathbf{x}_{e}\right)=\left[\begin{array}{ccc}
\eta_{1} \cos (\theta+\zeta) & 0 & \sin (\theta+\zeta) \\
\eta_{1} \sin (\theta+\zeta) & 0 & -\cos (\theta+\zeta) \\
0 & \eta_{1} / \sigma_{F} & \sigma / \sigma_{F}
\end{array}\right],
\end{gathered}
$$

and the auxiliary control input $\mathbf{u}_{a}$ is designed as,

$$
\mathbf{u}_{a}=-\mathbf{H}_{1}\left(\boldsymbol{\varphi}-\boldsymbol{\varphi}_{\text {ref }}\right)-\mathbf{H}_{2}\left(\dot{\boldsymbol{\varphi}}-\dot{\varphi}_{\text {ref }}\right)+\ddot{\varphi}_{\text {ref }} .
$$

Note that the control law (9) is invariant to the number of robots in the formation, and given $\sigma_{2}, \sigma_{3}, \ldots, \sigma_{n}$, no communication exchange between the robots is needed at runtime (in this sense, it can thus be regarded as decentralized).

\section{Case study: hexagonal formation of unicycles}

We evaluated the effectiveness of the trajectory tracking control law (9) on a type $(1,1)$ formation of six unicycles disposed at the vertices of a regular hexagon of side $d=3 \mathrm{~m}$. Since $n=6$, then $\gamma=\pi / 3$ and $r_{c}=d=3 \mathrm{~m}$. Moreover, $\left[\sigma_{2}, \sigma_{3}, \sigma_{4}, \sigma_{5}, \sigma_{6}\right]^{T}=$ $[1 / d, 1 /(\sqrt{3} d), 1 /(2 d), 1 /(\sqrt{3} d), 1 / d]^{T}$ and thus $\sigma_{F}=$ $1 /(2 d)=1 / 6 \mathrm{~m}^{-1}$. We chose the initial condition $\mathbf{x}_{e}(0)=[0,36,0,0,1 / 2,1]^{T}$ and selected the following proportional and derivative diagonal gain matrices: $\mathbf{H}_{1}=$ $\operatorname{diag}(16,6,2)$ and $\mathbf{H}_{2}=\operatorname{diag}(14,8,4)$. Finally, we defined the following elliptical reference trajectory for $\varphi(t)$,

$$
\boldsymbol{\varphi}_{\mathrm{ref}}(t)=\left[\mu_{\mathrm{M}} \cos (t), \mu_{\mathrm{m}} \sin (t), \frac{\pi}{4 \sigma_{F}}\right]^{T}, t \in[0,15] \mathrm{s},
$$




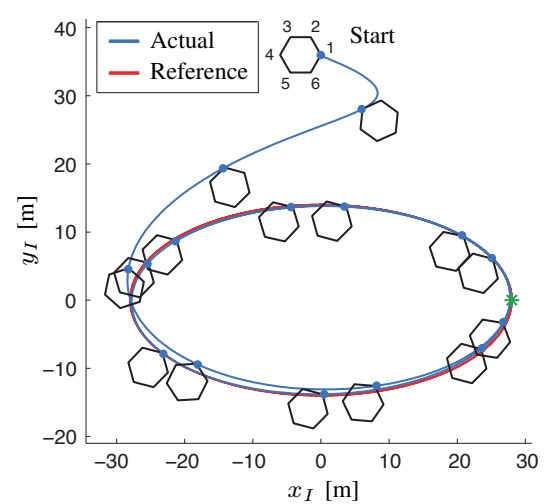

(a)

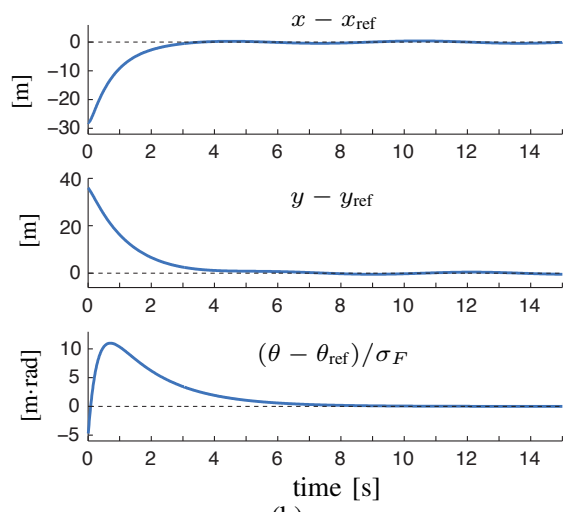

(b)

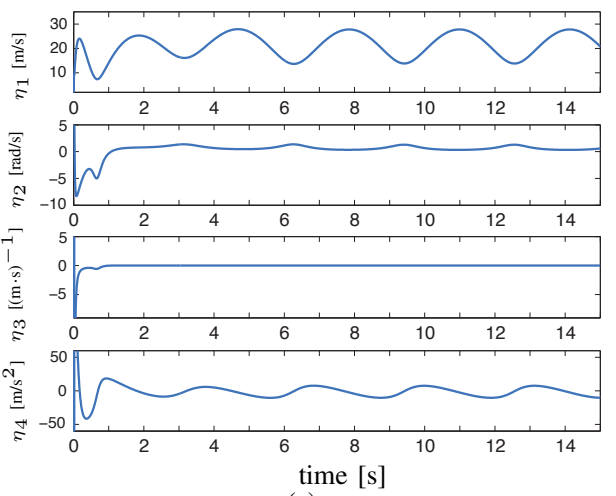

(c)

Fig. 5. (a) Trajectory of robot 1 of the hexagonal formation of unicycles (blue, actual; red, reference): to provide a time reference to the reader, the type $(1,1)$ formation is depicted every second; (b) Time evolution of the trajectory-tracking error $\boldsymbol{\varphi}(t)-\boldsymbol{\varphi}_{\text {ref }}(t)$; (c) Time history of $\eta_{1}(t)$ and $\mathbf{u}_{e}(t)$.

where $\mu_{\mathrm{M}}=28 \mathrm{~m}, \mu_{\mathrm{m}}=14 \mathrm{~m}$ are the lengths of the semi-major and semi-minor axes of the ellipse, respectively. Note that $\boldsymbol{\varphi}_{\text {ref }}(0)=[28,0,3 \pi / 2]^{T}$ (see the green star in Fig. 5(a)). Fig. 5(a) reports the trajectory of robot 1 (i.e. m-wheel 1) of the formation, Fig. 5(b) the time evolution of the trajectory-tracking error $\varphi(t)-\varphi_{\text {ref }}(t)$, and Fig. 5(c) the time history of $\eta_{1}(t)$ and $\mathbf{u}_{e}(t)$. Note that $\eta_{1}(t) \geq 1, \forall t \in[0,15] \mathrm{s}$, hence no singular rest configuration is crossed by the formation. A video illustrating the simulation results is available at the address reported below ${ }^{1}$.

\section{CONCLUSiOns AND FUture WORK}

In this paper, we have studied the mobility of distancebearing formations of unicycle robots and introduced a new classification, corresponding to two indices $\left(\delta_{m}, \delta_{s}\right)$, inspired by the taxonomy in [14].

This work is ongoing and we plan to extend our analysis in several directions. More general formations lacking dihedral symmetry and other inter-robot constraints (e.g. relative positions and heading angles) will be investigated. More involved wheel's and robot's models will be studied as well: in this respect, conventional off-centered orientable wheels and car-like robots, seem the most natural candidates. Finally, we would like to establish a link between the type of a formation and the (rigidity) properties of the weighted graph which defines the constraints between the robots.

\section{REFERENCES}

[1] J. Alonso-Mora, S. Baker, and D. Rus. Multi-robot formation control and object transport in dynamic environments via constrained optimization. Int. J. Robot. Res., 36(9):1000-1021, 2017.

[2] A. Farinelli, N. Boscolo, E. Zanotto, and E. Pagello. Advanced approaches for multi-robot coordination in logistic scenarios. Robot. Autonom. Syst., 90:34-44, 2017.

[3] G. Caprari et al. Highly Compact Robots for Inspection of Power Plants. J. Field Robot., 29(1):47-68, 2012.

[4] C. Belta and V. Kumar. Abstraction and Control for Groups of Robots. IEEE Trans. Robot., 20(5):865-875, 2004.

[5] K.-K. Oh, M.-C. Park, and H.-S. Ahn. A survey of multi-agent formation control. Automatica, 53:424-440, 2015.

[6] P. Tabuada, G.J. Pappas, and P. Lima. Motion Feasibility of MultiAgent Formations. IEEE Trans. Robot., 21(3):387-392, 2005.

\footnotetext{
${ }^{1}$ https://home.mis.u-picardie.fr/ fabio/Eng/Video/ MOBr_IROS $18 \cdot \mathrm{m} 4 \mathrm{v}$
}

[7] Z. Sun and B.D.O. Anderson. Formation feasibility on coordination control of networked heterogeneous systems with drift terms. In Proc. 55th IEEE Conf. Dec. Contr., pages 3462-3467, 2016.

[8] B.D.O. Anderson, C. Yu, B. Fidan, and J. Hendrickx. Rigid Graph Control Architectures for Autonomous Formations. IEEE Contr. Syst. Mag., 28(6):48-63, 2008.

[9] L. Krick, M.E. Broucke, and B.A. Francis. Stabilisation of Infinitesimally Rigid Formations of Multi-Robot Networks. Int. J. Control, 82(3):423-439, 2009.

[10] D. Zelazo, P. Robuffo Giordano, and A. Franchi. Bearing-Only Formation Control Using an SE(2) Rigidity Theory. In Proc. 54th IEEE Conf. Dec. Contr., pages 6121-6126, 2015.

[11] S. Zhao and D. Zelazo. Bearing Rigidity and Almost Global Bearing-Only Formation Stabilization. IEEE Trans. Automat. Contr., 61(5):1255-1268, 2016.

[12] G. Stacey and R. Mahony. The Role of Symmetry in Rigidity Analysis: A Tool for Network Localisation and Formation Control. IEEE Trans. Automat. Contr., 63(5):1313-1328, 2018.

[13] Z. Sun, S. Mou, B.D.O. Anderson, and C. Yu. Conservation and decay laws in distributed coordination control systems. Automatica, 87:1-7, 2018

[14] G. Campion, G. Bastin, and B. D'Andréa-Novel. Structural Properties and Classification of Kinematic and Dynamic Models of Wheeled Mobile Robots. IEEE Trans. Robot. Autom., 12(1):47-62, 1996.

[15] M. Egerstedt and X. Hu. Formation Constrained Multi-Agent Control. IEEE Trans. Robot. Autom., 17(6):947-951, 2001.

[16] W. Dong and J.A. Farrell. Cooperative Control of Multiple Nonholonomic Mobile Agents. IEEE Trans. Automat. Contr., 53(6):1434-1448, 2008.

[17] L. Consolini, F. Morbidi, D. Prattichizzo, and M. Tosques. Stabilization of a Hierarchical Formation of Unicycle Robots with Velocity and Curvature Constraints. IEEE Trans. Robot., 25(5):1176-1184, 2009.

[18] S. Zhao, D.V. Dimarogonas, Z. Sun, and D. Bauso. A General Approach to Coordination Control of Mobile Agents with Motion Constraints. IEEE Trans. Automat. Contr., 63(5):1509-1516, 2018.

[19] B. Thuilot, B. d'Andréa Novel, and A. Micaelli. Modeling and Feedback Control of Mobile Robots Equipped with Several Steering Wheels. IEEE Trans. Robot. Autom., 12(3):375-390, 1996.

[20] L. Consolini, F. Morbidi, D. Prattichizzo, and M. Tosques. On a Class of Hierarchical Formations of Unicycles and their Internal Dynamics. IEEE Trans. Automat. Contr., 57(4):845-859, 2012.

[21] W. Chung and K. Iagnemma. Wheeled Robots. In B. Siciliano and O. Khatib, editors, Springer Handbook of Robotics, chapter 24, pages 575-594. Springer, 2nd edition, 2016.

[22] L. Clavien, M. Lauria, and F. Michaud. Estimation of the instantaneous centre of rotation with nonholonomic omnidirectional mobile robots. Robot. Autonom. Syst., 106:47-57, 2018.

[23] R. Siegwart, I.R. Nourbakhsh, and D. Scaramuzza. Introduction to Autonomous Mobile Robots. MIT press, 2011.

[24] D.S. Bernstein. Matrix Mathematics: Theory, Facts, and Formulas. Princeton University Press, 2009.

[25] G.A. Korn and T.M. Korn. Mathematical Handbook for Scientists and Engineers: Definitions, Theorems, and Formulas for Reference and Review. Dover Publications, 2000. 